\title{
Ärztliche Qualität - im Dialog ausgehandelt
}

Die Diskussionen um die Qualität der medizinischen Versorgung begleiten uns auf Schritt und Tritt. In der Gesundheitspolitik, bei unseren Partnern und innerhalb der FMH. Vor allem bei den Ärztinnen und Ärzten ist das Thema alltäglich. Das Bemühen um Qualität hat etwas mit Haltung und Berufsethos zu tun und ist eine Grundvoraussetzung für die Ausübung unseres Berufes. Ausserhalb der Ärzteschaft wird in regelmässigen Abständen die Meinung geäussert, dass bezüglich medizinischer Qualität nichts geschehe und verbindliche Qualitätsstandards definiert werden müssten. Implizit wird dabei gelegentlich davon ausgegangen, dass mit dem Definieren irgendwelcher Normen und bürokratischer Erbsenzählerei die Qualitätsarbeit getan wäre.

Die FMH betrachtet Qualitätsarbeit als viel umfassender [1]. Unbestritten ist, dass zahlreiche Partner, allen voran die Patienten, Anspruch auf Qualität der ärztlichen Versorgung haben. Offensichtlich ist aber auch, dass bei einer Vielzahl von Anspruchsgruppen sich auch die Qualitätsvorstellungen deutlich unterscheiden. Daher muss es ein zentraler Aspekt bleiben, dass die angestrebten Ziele im Diskurs mit den Anspruchsgruppen, mit allen Anspruchsgruppen, verhandelt werden. Das Vorpreschen einzelner Partner, hier einseitig Direktiven festlegen zu wollen, ist unsinnig und kontraproduktiv. Im weiteren ist das Aushandeln kein einmaliger Akt, sondern muss angesichts der dynamischen Grösse Qualität kontinuierlich geschehen. Trotz des damit verbundenen Aufwands ist die FMH überzeugt, dass nur in einem solchen Prozess die Grundlage für eine sinnvolle Qualitätsarbeit liegen kann und hat wiederholt ihre Dialogbereitschaft signalisiert.
Daneben darf die Ärzteschaft auch immer wieder auf die Vielzahl von Aktivitäten hinweisen, die sie in ihrem Bereich seit Jahren zur Förderung der medizinischen Qualität durchführt. Wir leisten Qualitätsarbeit: in Qualitätszirkeln, im Einsatz von Fehlermeldesystemen, im Entwickeln und Implementieren von Praxisassessments und nicht zuletzt im Bereitstellen einer qualitativ hochstehenden Weiter- und Fortbildung. Es ist eine Mischung aus unzähligen Initiativen von der lokalen Basis bis hin zum internationalen Projekt, und alle dienen sie der Qualitätsförderung, wobei im Zentrum der ärztlichen Qualitätsarbeit stets die Bedürfnisse der Patienten stehen. Die Herausforderung ist zweifellos, dass diese zahlreichen Aktivitäten für die Öffentlichkeit und insbesondere für die Patienten sicht- und begreifbar werden.

Doch auch dies soll keine einseitige Deklaration sein. Es ist nur ein Hinweis darauf, dass die Ärzteschaft im Dialog mit den anderen Anspruchsgruppen viel zu bieten hat. Und hier schliesst sich der Kreis hin zum eingangs erwähnten, gewünschten Dialog: Welche sinnvollen gemeinsamen Ziele wollen wir uns für die Wahrung und Förderung einer medizinischen Versorgung von welcher Qualität setzen? Und was ist diese Qualität der Gesellschaft wert? Eine Frage, die nicht genug diskutiert werden kann ...

PD Dr. med. Ludwig T. Heuss, Mitglied des Zentralvorstandes, Ressortverantwortlicher DDQ

1 Peltenburg M, Kernen H, Schneider P, von Below GC, Waldis G, Vogel HA, et al. Qualität - ein Zusammenspiel aller Kräfte im ärztlichen Umfeld. Schweiz Ärztezeitung 2005;86(18):1055-9. 